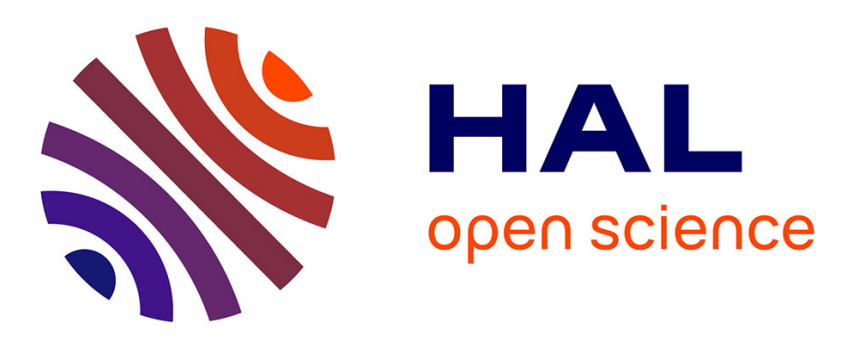

\title{
Des grands soirs aux beaux jours. La question environnementale peut-elle être encore porteuse d'utopies?
}

\author{
Chantal Aspe, Marie Jacqué
}

\section{- To cite this version:}

Chantal Aspe, Marie Jacqué. Des grands soirs aux beaux jours. La question environnementale peut-elle être encore porteuse d'utopies?. Education et Sociétés: Revue internationale de sociologie de l'éducation, 2016, Utopies et Education: des groupes en mouvement, 1 (37), pp.33-48. 10.3917/es.037.0033 . hal-01477587

\section{HAL Id: hal-01477587 \\ https://hal.science/hal-01477587}

Submitted on 18 May 2018

HAL is a multi-disciplinary open access archive for the deposit and dissemination of scientific research documents, whether they are published or not. The documents may come from teaching and research institutions in France or abroad, or from public or private research centers.
L'archive ouverte pluridisciplinaire HAL, est destinée au dépôt et à la diffusion de documents scientifiques de niveau recherche, publiés ou non, émanant des établissements d'enseignement et de recherche français ou étrangers, des laboratoires publics ou privés. 
ont aussi accompagné une recomposition du capitalisme qui en cherchant à désarmer cette critique a réussi non seulement à l'intégrer, mais aussi à s'en nourrir pour se renforcer (Boltanski \& Chiapello 1999).

Après avoir présenté comment les mobilisations des années 1970 ont construit un cadre théorique et militant à l'action écologiste, l'article aborde ce rapport dynamique entre idéologie et utopie (Manheim 1929) par l'analyse de plusieurs générations d'étudiants en environnement et de leur devenir professionnel. L'étude commence en 1992, date de création en France du premier Institut Universitaire Professionnalisé dans le domaine de l'environnement (IUP Environnement, Technologie et Société, IUP dans la suite du texte). Ces établissements (décret du 23 janvier 1992) associant des enseignements universitaires et professionnels recrutaient à $\mathrm{Bac}+1$ pour une formation de 3 ans et délivraient un diplôme d'Ingénieur-maître $(\mathrm{Bac}+4)$. Leur statut a été modifié à partir de 2006 pour intégrer ces cursus au cadre européen des LMD (Licence Master Doctorat). La période analysée s'étend, au fil des formations qui se sont succédé, jusqu'à l'actuel parcours d'un master en sciences de l'environnement. Le matériau repose sur plusieurs sources : la mémoire des auteures qui ont participé à la création et mise en œuvre de cet IUP à Marseille, les archives de travaux d'étudiants (rapports de stages et de missions), les annuaires d'anciens étudiants, les informations des sites Internet pour retracer leur devenir professionnel, des entretiens auprès d'un échantillon d'étudiants sortis récemment du parcours "Médiation scientifique en environnement" et d'autres dont nous avons eu la responsabilité comme stagiaires.

\section{S'éloigner de la société de consommation : autre part, ailleurs et autrement}

T a contestation écologiste et les mouvements utopistes nés avec le foisonnenérable qu'il y paraissait et que d'autres possibles étaient envisageables pour peu que l'on s'y mette ensemble" (Mathieu 2010, 16). Les mobilisations éparses des années 1970 ont en commun de déployer une nouvelle critique du capitalisme, dont l'enjeu est la dénonciation des rapports de domination. Au travail, dans la famille, à l'école, dans le couple, le syndicat, le parti... les luttes politiques des années 1970 ont remis en cause les fondements normatifs de la vie sociale par une critique systématique de ses rapports hiérarchiques (Damamme et al. 2008, Mathieu 2010).

À juste titre associée à cette mouvance de Mai 68, la critique écologiste comporte aussi une dimension propre : la remise en cause du mode de développement 
centré sur la croissance. Gorz, théoricien du mouvement d'écologie politique, propose à partir d'une lecture anti-productiviste de Marx, une critique du capitalisme à l'aune de la crise écologique. Pour lui, la seule façon de dépasser les contradictions et les crises successives de ce mode de développement est de sortir d'une vision productiviste. "La croissance économique, qui devait assurer l'abondance et le bien-être à tous, a fait croitre les besoins plus vite qu'elle ne parvenait à les satisfaire et abouti à un ensemble d'impasses qui ne sont pas économiques seulement : le capitalisme de croissance est en crise non seulement parce qu'il est capitaliste, mais aussi parce qu'il est de croissance" (Gorz 1975, 16).

La critique de la croissance porte sur les impasses économiques et écologiques auxquelles elle conduit les sociétés contemporaines et sur le rôle que joue le mythe d'une société d'abondance et de consommation comme instrument de pouvoir. L'apport d'intellectuels comme Illich $(1971,1973)$ ou Baudrillard (1970) sur la société de consommation a contribué à la construction de l'écologie politique. Illich dénonce le poids des institutions et de l'industrialisation des modes de vie sur l'asservissement des individus. "Un monopole radical s'établit quand les gens abandonnent leur capacité innée de faire ce qu'ils peuvent pour euxmêmes et pour les autres, en échange de quelque chose de mieux que peut seulement produire pour eux un outil dominant. Cette domination de l'outil instaure la consommation obligatoire, c'est-à-dire transforme l'individu en consommateur passif d'une production de masse que seules les grosses industries peuvent assurer. Finalement, même les besoins élémentaires ne peuvent plus être satisfaits hors commerce" (Illich 1973, 19). Pour sortir de ce système, il en appelle à la construction d'une société "conviviale", "qui donne à l'homme la possibilité d'exercer l'action la plus autonome et la plus créative, à l'aide d'outils moins contrôlables par autrui" (Illich 1971, 43). Cette quête de l'autonomie et de la liberté se fonde sur une remise en cause des institutions "porteuses de progrès" prétendant réaliser le bien-être des individus à leur place et dont la finalité reste pour Gorz et Illich une "extension des rapports marchands à tous les domaines de la vie individuelle et sociale et leur domination par les monopoles industriels, bancaires et étatiques" (Gorz 1975, 45).

Les luttes et les expérimentations sociales inspirées du foisonnement des années 1970 ont donné une portée concrète à ces écrits théoriques. Bien que minoritaire et marginal, le mouvement communautaire de retour à la terre a eu une portée politique majeure. Loin des formes instituées d'adhésions politiques ou syndicales, ces utopiens en mouvements proposent par leur parcours un autre rapport à l'engagement : autre part, ailleurs et autrement.

En ne retournant pas à l'usine ou au bureau, en refusant d'exercer des professions d'encadrement auxquelles ils étaient destinés, les utopiens communautaires ont quitté la ville, cherchant un lieu où pourrait prendre corps une société nouvelle, hors du temps capitaliste et de l'espace industriel, réinvestissant les 
lieux délaissés par le système dominant telles les zones de moyenne montagne (Ardèche, Lozère, Alpes-de-Haute-Provence, Cévennes).

À la suite des événements de 1968, environ 5000 personnes ont décidé de s'installer dans des zones affectées par l'exode rural pour fonder des communautés (Soulier 1978, Camels 1980, Vuarin 1982). Ces "immigrés de l'utopie" (Léger 1979, 50) venaient d'abord des grandes villes (Paris, Lyon, Marseille) et étaient pour la plupart étudiants ou jeunes actifs fraîchement diplômés. "La question de l'utopie se posait donc avec d'autant plus d'acuité : que cherchent ces 'urbains', ces enseignants, animateurs, travailleurs sociaux, membres des professions paramédicales, architectes qui en renonçant aux métiers et aux fonctions sociales pour lesquels ils ont été formés, se font éleveurs de chèvres ou de moutons, maraîchers, potiers ou tisserands ? Question qui en appelle logiquement une autre, celle de la nature de la crise sociale et culturelle dont le phénomène de retour est le symptôme" (Léger 1979, 47). Les analystes s'accordent sur le caractère socialement homogène de ce mouvement, porté par des acteurs issus majoritairement des classes moyennes et supérieures. Ils l'expliquent par l'expérience sociale commune de déclassement de cette génération de jeunes diplômés (Léger 1979, Lacroix 1981, Mauger 1999), mais aussi comme expression ponctuelle du phénomène général de positionnement politique des classes moyennes (Vuarin 1978).

L'enjeu fut de construire un ailleurs possible. L'espace rural abandonné par l'intensification agricole tint lieu de symbole. "Ces régions désertifiées pouvaient constituer un espace d'anticipation et d'expérimentation sociale parce que ces zones étaient tenues à l'écart des rapports économiques, sociaux et politiques qui structurent la 'société dominante'. C'était la friche (le 'désert') qui servait de support aux projets communautaires. Et, pour les intéressés, le fait de savoir que cet état d'abandon n'était pas le fruit de conditions géologiques ou climatiques, mais le produit direct de l'industrialisation capitaliste, donnait, d'emblée, une dimension 'politique' à ces projets" (Léger 1979, 48).

Investir ces espaces a permis l'expérimentation d'un autrement, d'une façon de produire, de consommer, d'échanger qui ne se situe plus dans des rapports marchands, mais dans l'expérience communautaire de la production, de l'échange, qui conduit à entrevoir les possibles d'une contre-société en marge, devenue conviviale. Cet autrement, est aussi la revendication de l'exemplarité. Loin des drapeaux rouges ou noirs, la visée politique est de faire tache d'huile en montrant la réalisation concrète d'une transformation sociale radicale.

Bien que minoritaires et pour beaucoup relativement éphémères, ces tentatives ont fortement marqué les formes de pensée de la société des années 1970 et le souvenir fantasmé des générations qui ont suivi. Elles ont aussi participé à la concrétisation d'une utopie écologiste en faisant du changement de vie, de l'expérimentation d'un autre mode de production et de consommation un de ses éléments constitutifs. Au début des années 1980, ces mobilisations contestataires 
semblaient déjà relever des illusions passées et bon nombre d'analyses concluaient à la fin des utopies. L'écologie politique s'est peu à peu intégrée dans le système : le parti des Verts est créé en 1984. Les enjeux écologiques s'effacent au profit d'une intégration à l'environnement, au marché (reprise de la croissance économique). Les espoirs portés par l'arrivée de la gauche au pouvoir en 1981 laissent place dès 1983 aux politiques de libéralisation de l'économie (Abdelal 2005). Le capitalisme s'internationalise, déplaçant l'enjeu de sa critique à une autre échelle.

\section{Un autre développement est possible}

E n 1992, vingt ans après la fondation du Programme des Nations Unies pour l'Environnement à Stockholm, se tint à Rio le second sommet de la Terre avec plus de 30000 délégués réunis pour proposer des solutions internationales aux enjeux de dégradation de l'environnement. Le dernier jour, alors que les solutions adoptées sont déjà décriées quant à leur efficacité environnementale et sociale (Doyle 1998, Sachs 1999, Vaillancourt 2002), Severrine Suzuki, une jeune Canadienne de 15 ans prend la parole sans que cela ait été prévu par le protocole. "Je suis seulement une enfant, mais je sais que nous faisons partie d'une famille de 5 milliards d'humains, en fait 30 millions d'espèces, et les gouvernements ne changeront jamais cela. Malgré ma colère, je ne suis pas aveugle et malgré ma peur, je n'ai pas peur de changer le monde comme je le sens. Dans mon pays, nous gaspillons tellement en jetant et achetant et nous ne partageons pas, même quand nous avons plus que suffisamment nous avons peur de perdre un peu de notre richesse" (Archives INA, 1992). Si la consécration de la notion de développement durable à Rio a marqué l'institutionnalisation de la question environnementale, elle a aussi été l'enjeu d'un nouveau projet utopiste : montrer qu'un autre mode de développement, plus égalitaire était possible.

\section{L'esprit de Rio}

Ce rapport militant au développement se retrouve chez les premiers étudiants ayant choisi l'IUP Environnement. Cette formation a représenté pour eux un "autre part", un lieu nouveau et innovant à l'université, permettant d'exprimer un rapport engagé à leurs études (quasi omniprésent dans les lettres de motivation obligatoires qui accompagnant les demandes d'inscription).

La création de l'IUP Environnement en 1992 fut une utopie en soi. Première formation universitaire dans ce domaine, elle fut aussi pionnière, car interdisciplinaire sciences sociales/ sciences de la nature. Loin de l'injonction contemporaine à l'interdisciplinarité, l'IUP fut d'abord un projet utopiste pour ceux qui l'ont 
porté en leur permettant d'ouvrir des horizons nouveaux dans les contenus ou la forme des enseignements. Sortir des découpages disciplinaires institués a nécessité d'inventer d'autres projets pédagogiques, qui ont pu aussi voir le jour parce que ses fondateurs s'y sont engagés sur la base de convictions partagées quant au rôle politique des pédagogies interdisciplinaires dans la formation des individus. La place occupée par les enseignements de sociologie (30\% de la totalité) dans cette formation "de" sciences et "en" sciences est sans doute un des indicateurs les plus remarquables du caractère innovant de cette formation.

Cette utopie pédagogique, qui aurait pu rester lettre morte, a rencontré son public en recherche d'ailleurs possibles. Au sein de l'IUP, les étudiants ont mis en pratique leurs engagements par la création d'associations, la rédaction et l'édition d'un mensuel, Mars'IUP, l'organisation d'activités collectives. Ils ont par leur dynamisme associatif créé des structures qui existent encore et qui à la fin des années 1990 représentaient de véritables innovations. Phénix, programme puis association d'aide au développement, initie ainsi dès 1994 des projets d'aide et de développement au Cambodge liant l'IUP et Médecins du Monde. Chaque année un petit groupe d'étudiants de deuxième année prend en charge ce programme, réalisant une analyse et un diagnostic d'un problème local (adduction d'eau, électrification, éducation) et assure le montage, le financement et la réalisation du projet au cours de leur année universitaire. Au fil des ans, plusieurs programmes ont vu le jour : Khivit au Cambodge, Karibo à Madagascar, Kim Lai au Vietnam, Dev'atan au Cameroun, Sagon au Bénin et au Togo. L'objectif premier de ces projets réside dans la défense de valeurs humanitaires : "Le projet Karibo est né d'un idéal humanitaire, au sens noble du terme, c'est-à-dire la recherche du bien de l'humanité, l'amélioration des conditions des hommes, de leur qualité de vie. S'investir dans le projet Karibo, c'est aussi répliquer aux incrédules que les valeurs tels la générosité, le partage, l'entraide existent et sont revendiquées par beaucoup" (Rapport Karibo 1995-1996, 4).

Les premiers projets de développement sont axés sur l'implication volontaire des étudiants. Leur réalisation signifie pour eux un engagement utopiste, car ils cherchent à mettre en pratique un autre mode de développement au service du bien-être des hommes et dans le respect de l'environnement. "Par-delà la diversité humaine, il nous paraît primordial de respecter les hommes en tant que peuple et en tant qu'entités individuelles, c'est-à-dire respecter aussi leur culture et l'environnement. De plus, nous pensons qu'une aide efficace ne peut s'inscrire que dans une perspective de long terme, en respectant et en favorisant l'autonomie des partenaires locaux" (Phénix, Charte, Principes fondamentaux). "Notre démarche est le développement durable, travailler dans le sens de l'autonomie et non de l'artisanat. Le but à moyen terme, c'est que les villages que nous aidons n'aient plus besoin de nous [...]. Pour cela, nous travaillerons à développer les moyens techniques permettant aux populations de se prendre en charge" (Phénix rapport de mission 1998). 
Ces projets de développement portés par les étudiants s'apparentent donc à une critique d'un "développement inégal" (Amin 1973) dont la réponse utopiste est de montrer par l'expérience qu'un développement, conciliant préservation de l'environnement et satisfaction des besoins humains, est possible s'il se fonde sur le respect et l'autonomie des peuples (Sachs 1980).

Cet engagement des premiers étudiants de l'IUP fait écho à un questionnement plus large des équilibres internationaux où le partage inégal des ressources et des richesses devient l'enjeu de revendications contestataires. Ces premiers "iupiens", comme ils se dénommèrent, ont choisi cette formation au regard de leurs convictions : la défense des droits de l'homme, la cause humanitaire et la défense de l'environnement. Ces trois thématiques recoupent ce qui, en gestation au début des années 1990, constitua une renaissance des mouvements contestataires, avec l'essor d'une nébuleuse altermondialiste (Sommier 2001, Agrikolianski et al. 2005). Si au cours des années 1970 le projet écologiste s'est construit dans un investissement du local, dans l'expérimentation communautaire au sein d'espaces ruraux marginalisés, son renouveau dans les années 1990 s'est élargi vers une contestation de la globalisation économique et de ses grandes institutions symboles : Fonds monétaire international, Banque mondiale, Organisation mondiale du commerce.

\section{D'une innovation sociale à une intégration technologique}

Si les premiers étudiants de l'IUP ont un rapport militant à la question environnementale, ils ont paradoxalement participé de son intégration en tant que domaine économique et professionnel. Pour s'insérer ils ont souvent dû créer leur poste de travail et militer au sein des entreprises et collectivités territoriales pour faire reconnaître l'importance d'un ingénieur environnement, d'un responsable qualité environnementale ou chargé de mission environnement, fonctions professionnelles qui, à cette période, n'existaient pas. Les compétences pluridisciplinaires dont ils sont porteurs les ont aussi amenés à défendre une approche critique et complexe des problèmes environnementaux. Si trouver un emploi était important pour eux, changer les pratiques à l'intérieur des organisations et des entreprises comptait tout autant.

Un suivi professionnel d'un échantillon de diplômés de l'IUP entre 1995 et 2008 (date du diplôme de la dernière promotion recrutée à l'IUP) montre deux périodes d'insertion professionnelle. De 1995 à 2002, leur parcours est à la fois plus militant et moins aisé que celui de la génération suivante. La période de 2003 à 2008 illustre l'intégration économique des enjeux environnementaux et le déploiement d'une croissance verte. Le tableau 1 synthétise une partie des informations sur le parcours professionnel de 380 étudiants -sur les 1384 ayant suivi le cursus- reconstitué à partir des archives de l'Association des étudiants en génie de l'environnement (ADEGE), des recherches par Internet via les réseaux sociaux et de relations professionnelles (linkedin et viadeo). 
Tableau I . Répartition de l'échantillon en fonction du domaine d'activité et de la période du diplôme

\begin{tabular}{|c|c|c|c|c|c|c|c|c|c|c|}
\hline \multirow{2}{*}{\begin{tabular}{|c|} 
Période \\
$1995-2002$ \\
\end{tabular}} & \multicolumn{2}{|c|}{ Environnement } & \multicolumn{2}{|c|}{$\begin{array}{c}\text { Protection et } \\
\text { gestion de la } \\
\text { nature }\end{array}$} & \multicolumn{2}{|c|}{\begin{tabular}{|c|} 
Traitement \\
des pollutions, \\
gestion des \\
risques
\end{tabular}} & \multicolumn{2}{|c|}{$\begin{array}{c}\text { Aide au } \\
\text { développement }\end{array}$} & Autres & \multirow{2}{*}{$\begin{array}{c}\text { Total } \\
223 \\
\end{array}$} \\
\hline & 109 & $(49 \%)$ & 46 & $(21 \%)$ & 24 & $(11 \%)$ & II & $(5 \%)$ & $33 \quad(14 \%)$ & \\
\hline $2003-2008$ & 46 & $(29 \%)$ & 31 & $(20 \%)$ & 67 & $(43 \%)$ & 5 & $(3 \%)$ & (5\%) & 157 \\
\hline Total & 155 & $(4 \mid \%)$ & 77 & $(20 \%)$ & 91 & (25\%) & 16 & $(4 \%)$ & $41 \quad(10 \%)$ & 380 \\
\hline
\end{tabular}

Répartition en fonction du domaine d'activité : Environnement (conseil, organisation, communication, gestion de services et HQE). Protection de la nature (gestion des espaces naturels, animation). Traitement technique des pollutions (traitement de l'eau, gestion des déchets, des risques industriels et nucléaires). Aide au développement (métiers de la coopération internationale). "Autres" regroupe ceux qui n'exercent pas dans l'environnement ou la protection de la nature.

Les diplômés de l'IUP entre 1995 et 2002 occupent majoritairement aujourd'hui ( $48 \%$ de notre échantillon) des postes de responsables de service dans les collectivités territoriales et 16\% d'entre eux ont créé leur entreprise ou bureau d'études, souvent en conseil ou communication (études d'impact, gestion de projets). Si la majorité a réalisé son insertion professionnelle dans des métiers en lien avec l'environnement, $14 \%$ exercent dans des domaines en lien avec un engagement politique, culturel ou éducatif. Ils ont épousé des métiers artistiques -metteur en scène, comédiens ou musiciens-, s'investissent dans l'éducation comme enseignants ou ont un engagement politique -élus de tendances écologistes dans des collectivités territoriales. Autant de métiers qui leur ont permis d'exprimer par la créativité, la pédagogie ou l'action locale, ce dont ils étaient porteurs : un désir de transformation sociale. La seconde génération s'insère davantage dans les entreprises privées, surtout elle n'exerce pas dans les mêmes secteurs. Si la première a réalisé son insertion professionnelle dans l'intégration de l'environnement aux politiques publiques, la seconde s'oriente vers des métiers techniques en lien avec le traitement des pollutions et la gestion des risques.

Cette deuxième génération a aussi choisi ces études plus en regard des débouchés. Pour eux, l'environnement est un secteur économique en croissance et l'IUP une formation "en environnement" dans un univers concurrentiel. Par exemple, sur le site de l'Onisep, parmi les 20 domaines proposés, "environnement, énergie, aménagement" est celui qui regroupe le plus de formations : 1436 à niveau Bac+3 et Bac+5. (<www.onisep.fr> en ligne le 25-08-2015).

L'environnement étant devenu un secteur économique, les problèmes écologiques sont moins des enjeux sociaux ou politiques que des défis technologiques. Le secteur des écoactivités a connu depuis une dizaine d'années une croissance exponentielle en termes d'emplois (croissance annuelle de 3,4\% en création d'emplois, nettement au-dessus de la moyenne, Thevenot \& Giestas 2012) ou en chiffre 
d'affaires pour les principales entreprises, Véolia et Suez (DGE 2009). La dynamique économique liée aux écoactivités est due à trois secteurs : le traitement des déchets, celui de l'eau et les énergies renouvelables avec une hausse historique de 25,5\% de croissance dans les écoactivités entre 2007 et 2008 (Randriambololona 2014). Ce secteur reste un des plus porteurs avec des taux de croissance positifs alors que le reste de l'économie stagne. Cette intégration économique s'est accompagnée d'une intégration idéologique des enjeux environnementaux. Le geste écocitoyen, devenu norme d'engagement, est soumis à la logique gestionnaire et technique de sa prise en charge ce qui le rend paradoxal. En participant à la gestion de l'environnement par leurs gestes, les individus s'engagent pour la protection de l'environnement et le font, la plupart du temps, dans une volonté de changement des formes de consommation et de production dominantes, mais ce même geste les enferme dans une logique gestionnaire qui perpétue ces formes d'organisation (Aspe \& Jacqué 2015).

\title{
Vers une utopie de l'autosuffisance
}

\begin{abstract}
A ux marges de ce mouvement d'intégration économique et idéologique des enjeux environnementaux, de nouvelles formes de résistance apparaissent. Depuis 2011-2012, un changement d'orientation à la fin de leur cursus universitaire s'observe chez des étudiants de master. Au lieu de s'insérer dans des métiers auxquels leur formation les destinerait (médiateurs en environnement, chargés de développement agricole, agronomes), ils expérimentent d'autres voies, qu'ils qualifient d'alternatives. Lors de stages, de voyages, de "woofing" (échanges au sein du réseau mondial de travail bénévole dans des fermes biologiques: World-Wide Opportunities on Organic Farms) entre deux périodes d'emplois, ils cherchent à découvrir des expériences et des façons de vivre en marge du modèle dominant de production et de consommation. Leur objectif est de construire et développer leurs propres projets s'appuyant sur l'idée que la multiplication et l'agrégation des alternatives rendent une transformation sociale envisageable. Ces nouveaux projets utopistes interrogent : quels sont leur portée contestataire et leur lien avec les mouvements passés? S'agit-il d'un renouveau de l'utopie communautaire des années 1970 ? Ces expériences s'appuient-elles comme les précédentes sur une critique radicale du mode de production? Comment sont-elles mises en œuvre par ceux qui s'en revendiquent?
\end{abstract}

\section{La valorisation de l'autonomie : faire ensemble pour soi}

L'analyse de dix entretiens exploratoires auprès de six étudiants diplômés du master médiation scientifique en environnement, obtenu depuis 2010, et de quatre étudiants diplômés de master d'agronomie dévoile un point commun : la référence 
centrale à l'autonomie dans leur discours. En allant travailler chez de petits producteurs en circuits courts, en agriculture paysanne et agroécologie, dans des filières de production artisanale comme la transformation de plantes médicinales, en suivant des stages d'autoconstruction d'habitat ou d'énergie renouvelable, leur objectif est d'acquérir savoir-faire et techniques permettant de s'extraire d'une dépendance matérielle vis-à-vis du système dominant : "J'ai fait le choix de venir vivre ici pendant six mois, pour voir et apprendre, si je peux vivre comme un paysan. C'est difficile, mais on fait tout et c'est ça qui est merveilleux. Pour moi, l'autonomie c'est essentiel, c'est vital et c'est ça que je recherche ici, apprendre à être autonome" (H. 25 ans, Ingénieur agronome). "Je suis partie pratiquement un an à l'étranger pour voir si c'était possible de vivre sans être dépendante du système, d'arriver à vivre sans lui, en dehors de lui" (F. 23 ans, master médiation). "L'idée que je défends c'est on ne combat pas le système en voulant le détruire, mais en construisant sans lui. Il y a pas mal d'alternatives qui naissent dans tous les domaines et ça plus il y en aura plus ça déstabilisera le système” (H. 26 ans, master agronomie).

Pour conquérir cette autonomie, les étudiants interrogés valorisent l'acquisition de savoir-faire et la fréquentation de réseaux ou ateliers d'échange. Dans ces espaces, multipliés depuis, s'opère une forme de socialisation politique dont l'enjeu revendicatif porte sur l'échange non marchand comme moyen de détournement du système dominant. un de ces ateliers présente ainsi sa démarche : "Le principe de l'atelier est que celui qui détient un savoir ne l'utilise pas contre quelqu'un ou pour lui tout seul, mais puisse au contraire le partager. Apprendre, c'est aussi comprendre et démystifier la mécanique. L'objectif premier est de rendre chaque adhérent le plus autonome possible face à son véhicule en lui apprenant à l'entretenir afin d'éviter les urgences et les pannes. En apprenant comment fonctionne une automobile, sans devenir un spécialiste, on en devient beaucoup moins dépendant. Cela permet de privilégier l'être humain à son véhicule" (Valence Atelier Libre, www.vatelier.fr/?page_id=27, en ligne le 25-06-2015).

L'apprentissage par les savoir-faire et non les savoirs théoriques renverse leur rapport hiérarchique dominant de l'ordre des savoirs et celui des modes de leurs transmissions. La portée utopiste de cet "âge du faire" (Lallement 2015) réside dans la rupture opérée dans le rapport au travail qui doit être guidé par la recherche du plaisir dans un cadre non contraignant.

Le parallèle entre ces expériences collectives vécues dans les ateliers d'échanges ou la réalisation d'un projet alternatif et les communautés utopistes des années 1970 peut sembler évident. Elles diffèrent cependant sur un point central : pour les communautés il s'agissait de faire pour être ensemble, aujourd'hui, l'enjeu est davantage d'être ensemble et de faire pour soi. "L'important aujourd'hui dans ma démarche, c'est de repasser par un système d'entraide. Dépendre des autres, mais pas par le biais du système marchand et monétaire. L'idée, c'est j'ai un ami qui sait faire ça, tu me le fais, en contrepartie je te donne autre chose, on échange, ça 
me plaît cette idée, c'est plus humain pour moi et finalement chacun est content" (H. master agronomie, 23 ans). "Oui à travers l'atelier nous avons rencontré beaucoup de gens, mais la réalisation de notre projet, le noyau c'est mon frangin et moi. Après on peut intégrer quelques personnes, on a un pote luthier qui peut faire un atelier construction de guitare et encore quelques personnes. Mais on n'a pas envie non plus d'être 15 000" (H. master médiation, 22 ans). "Ce que je trouve bien ici [dans l'atelier] c'est qu'il y a des gens qui à la fois exercent leur activité professionnelle et d'autres qui viennent pour apprendre, en fait c'est le fait que chacun y trouve son compte dans un échange désintéressé" (F. master médiation, 22 ans). "Le projet c'est monter une Gaec avec des copains de fac, on verra si ça se fera, mais pour le moment on y tient. Mais si je n'arrive pas à le faire en collectif, j'essaierai quand même avec ma copine" (H. master agronomie, 26 ans).

La recherche d'autonomie n'a pas la même portée politique pour les utopiens d'aujourd'hui et ceux d'hier. L'autonomie des années 1970 était synonyme d'autogestion et le cœur de la démarche n'était pas la capacité à produire pour soi-même, mais l'organisation différente qu'impliquait un changement de mode de production, l'ensemble des rapports sociaux institués était questionné. Aujourd'hui, l'autonomie renvoie à une recherche de maîtrise de la technique et des savoir-faire comme maîtrise de son projet de vie. Si les interrogés déclarent tous vouloir s'investir dans une alternative visant l'autonomie, aucun n'a pour l'instant concrétisé cet engagement, il apparaît plus comme un but qui donne sens aux acquisitions actuelles. En attendant, rien n'empêche de rester chez les parents pour préparer tranquillement son projet, la famille n'étant plus un espace de socialisation et d'autorité à combattre, mais un refuge de ressourcement. L'inscription dans un projet collectif passe par l'insertion au sein de réseaux, l'expérience collective étant en définitive plus réticulaire que communautaire.

Ces expériences alternatives peuvent être rapprochées de formes contemporaines d'engagement politique, centrées sur l'individu et la réalisation de soi à travers le projet collectif. La question de l'individualité et de la reconnaissance de soi devient un élément clé des mobilisations politiques, qui, par les actes individuels et la revendication d'une multiplication d'expériences, signifient l'engagement au

politique. L'individualisme n'est pas la cause d'un déclin supposé de l'intérêt pour la chose publique, mais la source d'une diversification et d'une multiplication des formes de l'engagement (Ion 2014). Il nécessite peut-être aussi une reconstruction politique du sujet qui dépasse les cadres de son rôle d'individu limité aux fonctions de consommateur.

\section{La consommation, une nouvelle arme de la critique?}

Face à la crise écologique, les utopiens développent aussi une philosophie critique et pratique de la consommation : décroissance, consommation raisonnée 
et raisonnable (biologique, éthique, écologique), simplicité volontaire, recours au troc, à l'échange, à la création de monnaies locales, de logiciels libres, regroupements d'achats en coopératives, etc. (Frère \& Jacquemain 2013, Bard et al. 2014). Ces pratiques sont analysées au regard de leur portée critique et contestataire vis-à-vis des promesses non tenues de la société de consommation et comme résistance à l'emprise croissante d'un mode de développement consumériste (Dobré 2002, Manier 2012, Maresca \& Dujin 2014). Elles font aussi écho à des formes plus radicales de changement de modes de vie en conformité avec une certaine frugalité -habitat léger, autoproduction totale (Pruvost 2013). La contestation contemporaine invite chacun à transformer son quotidien pour à la fois agir sur le tout et montrer par l'exemplarité qu'il est possible de vivre dans une "simplicité volontaire" (Aries 2010).

La recherche d'un mode de vie le plus écologique possible ne signifie pas, pour les interrogés, sortir du système de consommation dominant, mais faire de l'acte de consommer le moyen et l'objet de sa critique. "C'est sûr que dans ce projet, ce qui est important c'est aussi de valoriser un autre mode de vie, de montrer qu'il est possible de vivre bien, heureux sans forcément toujours acheter plus" (F. 23 ans, master médiation). "Quand tu fais du petit éolien de toute façon tu n'as pas le même rapport à la consommation d'énergie. Forcément tu ne peux pas produire autant qu'avec du nucléaire ou même du grand éolien, du coup ça nécessite aussi de s'adapter, de vivre différemment, d'être un peu plus économe" (H. 22 ans, master médiation). "L'idéal, c'est sûr, ce serait d'avoir un mode de vie en accord avec mes principes. Économiquement, je n'y arrive pas toujours, je vais plus chez Lidl que chez Biocoop. Je fais attention à ce que je consomme, j'essaie d'acheter local" (F. 25 ans, master agronomie).

Pour les protagonistes interrogés, changer son mode de consommation passe par le développement de pratiques d'autoproduction et de fabrication artisanale. Contre le grand éolien, il est possible de produire soi-même son électricité en fabriquant du mini-éolien ou de faire soi-même ses vêtements et chaussures, son jardin et potager. Ces pratiques alternatives permettent de maintenir une consommation écologiquement responsable dans une conjoncture où ce qui était hier vu comme des solutions écologiques locales (l'éolien, le solaire, le bio, etc.) sont devenues des secteurs industriels à la production internationalisée. La contestation écologiste doit composer avec un capitalisme devenu "vert" dont l'essor est promulgué comme la seule réponse à une crise écologique majeure, celle du changement climatique. La récente "loi sur la transition énergétique pour une croissance verte", du 17 août 2015 se présente comme devant "faire émerger un nouveau modèle de société en libérant les initiatives". Les innovations technologiques y tiennent une place centrale, favorisant la croissance économique de secteurs tels le bâtiment, la production d'énergies propres en $\mathrm{CO} 2$, dont le nucléaire. La réponse contestataire des utopiens de l'échantillon est de défendre la valeur de formes plus artisanales 
et locales de consommation écologiquement responsable. La consommation guide la production : en décidant de consommer autrement -pas nécessairement moins- j'agis sur les modes de production. "Pour moi l'autoconstruction peut casser la récupération de l'environnement par le système. Le système capitaliste s'est adapté, par le tri des déchets, le développement durable. L'autoconstruction peut un peu plus le saper, parce qu'on est vraiment dans l'échange de savoirs, pas dans le brevet [...] ça permet aussi de produire en fonction de ses besoins et de ne pas être dans une course effrénée à la consommation" (H. 22 ans, master médiation). "C'est vrai que je me reconnais dans toutes les alternatives qui existent. En ce moment il y a Alternatiba qui tourne, ils vont passer à Marseille. Je me reconnais complètement dans cette démarche de valoriser [...] la façon dont on peut vivre différemment pour freiner le capitalisme, même celui qui prétend faire le bien de l'humanité" (H. 25 ans, master médiation).

Le mouvement Alternatiba (www.alternatiba.eu), mis en lumière au moment de la COP 21 de décembre 2015, relaie un ensemble de solutions alternatives du même ordre que celles présentées dans les entretiens : choisir une alimentation écologique issue de l'agriculture paysanne, soutenir les coopératives, les AMAP (Association pour le maintien d'une agriculture paysanne), faire du jardinage collectif, favoriser une consommation moins énergivore et participer à sa production en construisant du mini-éolien et des installations solaires, adhérer à des systèmes bancaires coopératifs, etc. Lélément fédérateur est de faire de l'acte de consommation un moyen de pression sur le système économique. Choisir sa consommation et participer à la satisfaire en produisant par soi-même ou par l'intermédiaire de groupes d'autoconstruction/production représente la voie concrétisant "une société plus agréable à vivre, plus solidaire, juste et humaine" (Alternatiba 2015). L'idée n'est pas de prôner l'arrivée révolutionnaire d'un grand soir, mais de profiter et de se construire de beaux jours.

Agir par et sur sa consommation permet à la nouvelle génération d'utopiens un aménagement de ses modes de vie, conciliant valeurs revendicatives et insertion dans une société de consommation à laquelle il leur est difficile d'échapper. Un des moyens d'agir sur soi, de se contrôler est d'aller trouver des exemples dans des sociétés en développement fonctionnant sur l'autosuffisance. À la sortie de leurs études, certains étudiants interrogés (4 sur 10) ont fait le choix de partir à l'étranger, d'acquérir une expérience autre part. "Je cherche dans mes voyages des choses alternatives, par rapport au mode de vie occidental, c'est-à-dire basé sur le capital, la croissance économique, etc., donc voir qu'il existe d'autres modes de vie, des solutions, des gens qui vivent autrement, ça marche, montrer qu'il y a des exemples qui marchent bien" (H. 24 ans, master agronomie).

La référence quasi unanime des étudiants à des personnalités comme Pierre Rabhi ou Vandana Shiva (évoqués dans 8 des 10 entretiens) témoigne de leur adhésion à la pensée d'une nouvelle "sobriété heureuse" (Rahbi 2010). L'un et l'autre 
défendent les modèles traditionnels d'agriculture paysanne contre leur destruction par les logiques agro-industrielles. L'action sur la consommation, par une recherche d'autosuffisance, est là encore centrale. La communauté paysanne idéalisée réaliserait cette sobriété heureuse et offre un point d'horizon aux expériences alternatives et utopistes, mais bien loin de celui des années 1970. S'il valorise une recherche d'autonomie vis-à-vis du mode de production dominant, la question des rapports sociaux de domination, internes aux formes communautaires traditionnelles, n'est pas posée. La sobriété heureuse pourrait alors rapidement "transformer une mentalité 'économe' en un accessoire d'une politique étatique de pénurie, à l'occasion de laquelle seraient expérimentés de nombreux mécanismes d'asservissement et d'obédience, ceci en toute bonne conscience "écologiste"' (Duclos 2009, 306).

\section{Conclusion}

La crise écologique contemporaine a constitué, depuis les années 1970, un puissant vecteur de remise en cause d'un développement industriel centré sur la croissance et le productivisme. Les mouvements de l'écologie politique radicale et les utopies communautaires ont construit une critique globale du système économique et des rapports sociaux, faisant de la question environnementale un enjeu de transformation sociale et économique. L'intégration progressive de cette critique et des revendications qu'elle porte s'est faite dans une dynamique entre récupération idéologique et ajustement utopiste (Ricœur 1984). Au cours des années 1990, l'individualisation croissante des modes de vie et l'amplification des solutions individuelles ont produit un réajustement des utopies environnementales. La portée politique de la pédagogie et la formation citoyenne sont présentées comme les principaux moteurs de transformation sociale. Les prétentions du système économique libéral à résoudre les problèmes écologiques s'expriment au cours de cette période par une intégration idéologique des individus à travers l'adoption de pratiques écocitoyennes.

Les alternatives écologistes développées aujourd'hui autour des choix et modes de consommation peuvent être lues comme une réponse utopiste à la récupération idéologique des revendications environnementales par un capitalisme devenu écologiquement viable. Elles ne portent pas de volonté de bouleverser les rapports sociaux, mais cherchent par l'arrangement des modes de vie et l'acte de consommation à construire un équilibre responsable entre environnement et développement économique. Contrairement aux utopies des années 1970 où l'expérimentation communautaire du changement de vie se nourrissait d'une critique théorique du mode de production capitaliste et des rapports sociaux qu'il conditionne, les alternatives actuelles ne s'inscrivent pas dans un projet politique de transformation sociale. Elles révèlent un désir d'engagement de cette nouvelle génération à la recherche d'autres cadres pour l'action collective. L'intérêt 
renouvelé pour des auteurs comme Gorz ou Illich par la publication de nombreux ouvrages qui leur sont consacrés (Fourel 2012, Dufoing 2012, Caillé \& Fourel 2013, Gollain 2014) laisse entrevoir les possibilités de donner un fondement politique et théorique à ces utopies alternatives.

\section{Références bibliographiques}

ABDELAL R. 2005 "Le consensus de Paris : la France et les règles de la finance mondiale”, Critique internationale-28, 87-115

AGRIKOLIANSKI E., FILLIEULE O. \& MAYER N. 2005 L'altermondialisme en France. La longue histoire d'une nouvelle cause, Paris, Flammarion

ALTERNATIBA < www.alternatiba.eu> en ligne le 31-08-2015

AMIN S. 1973 Le développement inégal. Essai sur les formations sociales du capitalisme périphérique, Paris, Éditions de Minuit

ARIÈS P. 2010 La simplicité volontaire contre le mythe de l'abondance, Paris, La Découverte

ASPE C. \& JACQUÉ M. 2012 Environnement et société. Une analyse sociologique de la question environnementale, Paris, Éditions FMSH / QUAE

ASPE C. \& JACQUÉ M. 2015 "From activism to alienation: the paradoxes of being an eco-citizen", Journal Of Harmonized Research in Management-1(2), 111-121

BARD C., BERTIN G. \& GUILLAUD L. dir. 2014 Figures de l'utopie. Hier et aujourd'hui, Rennes, PUR

BAUDRILLARD J. 1970 La société de consommation, Paris, Gallimard

BOLTANSKI L. \& CHIAPELLO È. 1999 Le nouvel esprit du capitalisme, Paris, Gallimard

CAILlÉ A. \& FOUREL C. dir. 2013 Sortir du capitalisme. Le scénario Gorz, Paris, Éditions Le Bord de l'eau

CALMELS G. 1980 Les nouvelles installations agricoles dans les Alpes de Haute-Provence, Rapport, Sisteron, Bureau d'Accueil et d'Information des Alpes du Sud

DAMAMME D., GOBILLE B., MATONTI F. \& PUDAL B. dir. 2008 Mai Juin 68, Paris, Éditions de l'Atelier

DGE (Direction Générale de l'Entreprise) 2009 "Eco-Industries : la croissance d'une filière", Cahiers Industrie-137, Paris, Ministère de l'Économie et des Finances

DOBRÉ M. 2002 L'écologie au quotidien. Éléments pour une théorie sociologique de la résistance ordinaire, Paris, L'Harmattan

DOYLE T. 1998 "Sustainable development and Agenda 21: the secular bible of free markets", Third World Quaterly-19(4), 771-786

DUCLOS D. 2009 La notion de mode de vie est-elle encore adéquate pour parler des changements à venir ?, in Dobré M. \& Juan S. dir. Consommer autrement. La réforme écologique des modes de vie, Paris, L'Harmattan, 305-309

DUFOING F. 2012 L'écologie radicale, Paris, Infolio

FOUREL C. dir. 2012 Gorz, Un penseur pour le XXI siècle, Paris, La Découverte

FRÈRE B. \& JACQUEMAIN M. 2013 Résister au quotidien ? Paris, Presses de Sciences Po 
GOLLAIN F. 2014 André Gorz. Pour une pensée de l'écosocialisme, Paris, Le Passager clandestin GORZ A. 1975 Écologie et politique, Paris, Galilée, Le Seuil

ILLICH I. 1971 Une société sans école, Paris, Le Seuil

ILLICH I. 1973 La convivialité, Paris, Le Seuil

ION J. 2014 S'engager dans une société d'individus, Paris, Armand Colin

LACROIX B. 1981 L'utopie communautaire. Histoire sociale d'une révolte, Paris, PUF

LALLEMENT M. 2015 L'âge du faire. Hacking, travail, anarchie, Paris, Le Seuil

LÉGER D. 1979 "Les utopies du 'retour", Actes de la recherche en sciences sociales-29, 45-63

MANHEIM K. 1929 [1956] Idéologie et Utopie. Une introduction à la sociologie de la connaissance, Paris, Librairie Marcel Rivières

MANIER B. 2012 Un million de révolutions tranquilles. Travail, Argent, Habitat, Santé, Environnement. Comment les citoyens changent le monde, Paris, Les Liens qui libèrent

MARESCA B. \& DUJIN A. 2014 "La transition énergétique à l'épreuve du mode de vie", Flux 2(96), 10-23

MATHIEU L. 2010 Les années 70, un âge d'or des luttes? Paris, Textuel

MAUGER G. 1999 “En France, trente ans après, comment analyser Mai 68 ?”, Scalpel-4(5), $169-177$

PRUVOST G. 2013 "L'alternative écologique. Vivre et travailler autrement”, Terrain-60, $36-55$

RABHI P. 2010 Vers la sobriété heureuse, Paris, Actes Sud

RANDRIAMBOLOLONA C. 2014 "Les éco-activités et l'emploi environnemental en 2012”, Chiffres et statistiques-523, Commissariat Général au Développement Durable

Rapport Karibo 1995-1996, Le devenir du projet Karibo Madagascar, Université de Provence, archive privée

RICOEUR P. 1984 "L'idéologie et l'utopie : deux expressions de l'imaginaire social”, Autretemps, Les Cahiers du christianisme social-1(2), 53-64

SACHS I. 1980 Stratégie de l'écodéveloppement, Paris, Éditions ouvrières

SACHS W. 1999 Planet dialectics. Explorations in environment and development, Londres, Zed Books

SOMMIER I. 2001 Les nouveaux mouvements contestataires à l'heure de la mobilisation, Paris, Flammarion

SOULIER A. 1978 "Les nouveaux occupants de l'espace rural. Contribution à l'étude des changements économiques et sociaux de l'arrière pays languedocien : exemple des Causses et des Cévennes lozériennes", Économie méridionale-26(100), 45-49

THEVENOT M. \& GIESTAS K. 2012 Les métiers verts, Dossier documentaire, Paris, Centre d'Études et de Recherches sur les Qualifications (CEREQ)

VAILLANCOURT J.-G. 2002 “Action 21 et développement durable”, Vertigo-3(3), en ligne le 18-02-2016 <http://vertigo.revues.org/4172s>

VUARIN R. 1982 Le recours à la terre. Essai de compréhension de la structure et explication de la genèse du mouvement contemporain d'installation à la terre de non-agriculteurs, Thèse de $3^{\text {e }}$ cycle en sociologie, Université de Provence

VUARIN R. 1978 Formes socio-économiques et déterminants idéologiques du phénomène de "retour à la terre", Rapport de Recherche, Aix-en-Provence, Laboratoire de sociologie de l'industrialisation et du développement, Université de Provence 